\title{
Report on the 18th Annual Land O'Lakes Bioanalytical Conference
}

\author{
Erik C Burns*,1, Raj Dhodda ${ }^{2}$, Stephanie Pasas-Farmer ${ }^{3}$, Eric Fluhler ${ }^{4}$, Randall Guthrie ${ }^{5}$, \\ Patrick Bennett ${ }^{6}$ \& Qin C Ji ${ }^{7}$ \\ ${ }^{1}$ University of Wisconsin, 777 Highland Ave., Madison, WI 53705, USA \\ ${ }^{2}$ AbbVie, North Chicago, IL 60064, USA \\ ${ }^{3}$ NanoMedical Systems, Austin, TX 78774 USA \\ ${ }^{4}$ Bioanalytical Compliance Associates, Groton, CT 06340, USA \\ ${ }^{5}$ Pyxant Labs, Colorado Springs, CO 80907, USA \\ ${ }^{6}$ PPD, Richmond, VA 23230, USA \\ ${ }^{7}$ Bristol-Myers Squibb, Princeton, NJ 08540, USA \\ * Author for correspondence: erik.burns@wisc.edu
}

The 18th Annual Land O'Lakes Bioanalytical Conference, titled 'Cutting-Edge Bioanalytical Technologies and Concepts - Issues, Solutions and Practical Considerations for Applications in Novel and Emerging Modalities', was held 10-13 July 2017 in Madison, WI, USA. This Land O'Lakes Conference is presented each year by the Division of Pharmacy Professional Development within the School of Pharmacy at the University of Wisconsin-Madison (USA). The purpose of this conference is to provide an educational forum to discuss issues and applications associated with the analysis of xenobiotics, metabolites, biologics and biomarkers in biological matrices. The conference is designed to include and encourage an open exchange of scientific and methodological applications for bioanalysis. This report summarized the presentations at the 18th Annual Conference.

First draft submitted: 17 January 2018; Accepted for publication: 1 February 2018; Published online: 10 April 2018

Keywords: bioanalysis $\bullet$ drug development $\bullet$ immunoassay $\bullet$ LC/MS technology $\bullet$ method validation $\bullet$ nanoparticles - professional development $\bullet$ regulatory guidance

The 18th Annual Land O'Lakes Bioanalytical Conference brings together an international group of bioanalytical scientists with the express intent of discussing cutting-edge science for small and large molecule bioanalysis in a relaxed atmosphere that promotes professional networking. It provides an educational forum with presentations by leaders from pharmaceutical and biotechnology industry, contract laboratories, vendors, consultants, and regulatory affairs authorities.

The success of the program is due to the Planning Committee, which consists of scientists involved with bioanalysis on a daily basis, and their ability to identify timely topics and presenters who are experts in the field. Specific objectives for the conference and a list of the Planning Committee members can be found online [1].

On Monday evening, the conference opened with dinner and a presentation by Mary Cheni Kwok, Managing Partner and Founder, Linear Dreams (CA, USA) that focused on the therapeutic promises and financial reward associated with cancer immunotherapy.

\section{Conference presentations}

Conference Chair, Raj Dhodda (AbbVie, IL, USA), opened the annual meeting by providing insights into key trends of emerging biologics with innovations that flow from assets such as immunotherapies, antibody-drug conjugates (ADCs), and gene and cell therapies. The first plenary session focused on integrated bioanalytical strategy from drug discovery to development. The opening presentation by Diego Cortes (PPD Laboratories, VA, USA) focused on the application and advantages of hybrid bioanalysis approaches to Monoclonal antibodies (mAbs), bispecifics and ADCs, which were effectively supported by relevant case studies and work flows on affinity capture method development approaches followed by method validation data. Cortes concluded that approaches using 
generic peptides would help accelerate early development go-no-go decisions by enabling pharmacokinetic (PK) analysis across animal species and candidates molecules with minimal effort in method development. The second presentation by Jennifer Postelnek (Brisol-Myers Squibb, NJ, USA) described how to drive to a decision on the approaches during discovery to early development. The key factors emphasized were the quality of reagents and the timeliness of their availability. With several case studies she walked through the derivation of strategy with relevance to competing and noncompeting antibodies and success of implementation of those strategies for developing a total assay for soluble targets. Postelnek concluded that both LBAs and LC-MS methods have their limitations; however, due to their orthogonal nature they complement each other and having both methods in the development increases overall success. The third presenter, Linzhi Chen (Boehringer Ingelheim Pharmaceuticals, CT, USA), led a discussion on immunogenicity assessment presenting two immuno-capture approaches (drug or a mouse mAbs as capture reagent) to detect pre-existing antidrug antibodies and polyclonal antibodies in blank human plasma. He emphasized that either drug or mAb could be used as immuocatpure reagent; using mAb eliminated potential drug interference issues. Both approaches gave similar results with $\mathrm{mAb}$ capture reagent being less sensitive. The challenges and solutions for method development and validation of the hybrid quantitative assays for ADCs was presented by Leo Kirkovsky (Pfizer, CA, USA). The biotransformation of calicheamicin as the payload was discussed together with development of hybrid methods for ADCs with calicheamicin. He emphasized the importance of characterizing the assay with individual or enriched drug to-antibody ratios (DARs) to assess if there is any bias for DARs in the assay. He concluded that during assay validation and performance, the focus should predominately on the generating a robust assay and the acceptance criteria may be relaxed if warranted. The plenary session concluded with Violet Lee (Genentech, CA, USA) sharing information on hybrid immunoaffinity LC-MS/MS for bioanalytical PK strategies for the next-generation of ADCs and Stephanie Pasas-Farmer (NanoMedical Systems, TX, USA) discussing the potential future landscape and challenges/opportunities for the bioanalytical industry.

Tuesday afternoon's session on nanoparticle therapeutics and cutting-edge bioanalytics began with Laura SeppLorenzino (Alnylam Pharmaceutical, MA, USA) discussing hepatocyte-targeted investigational RNAi therapeutics. Although these molecules have the potential of being powerful and life changing therapeutics, natural RNA molecules are not very 'druggable' for multiple reasons such as immuno-stimulation and instability in biological matrices. For the molecules discussed, additional liver exposure specificity has been achieved in the conjugation process through their Enhance Stabilization Chemistry. These hepatocyte-targeted Enhance Stabilization ChemistryGalNAc platforms have demonstrated favorable emerging profiles compared with competing oligo platforms with multiple human POCs studies on going and many others in planning stages. Next Mark Spengler (Chimera Biotec, Dortmund, Germany) introduced a platform for biologics and biomarkers, imperacer. Although, focusing on the determination of large molecule biotherapeutics and biomarkers, imperacer differs from High Sensitivity ELISA and Samoa by employing antibody-DNA conjugates that enable exponential signal amplification by PCR:DNA. The technique also overcomes current challenges in bioanalysis of large molecules. The third speaker, Dan Norris (Ionis Pharmaceuticals, CA, USA), discussed the development of second-generation antisense oligonucleotide and the bioanalytical challenges his team has encountered. ASOs have very specific properties that at sometimes are the same and other times different than their small molecule and biologics drug counterparts. Although, ASOs are distributed all over the body in tissues (except for the brain), the plasma levels are extremely low in comparison. The preclinical tissue concentrations are instrumental in driving the clinical dosing discussions and decisions. To help the profile of the preclinical exposure of ASOs, the bioanalyst must develop multiple assays due to the wide variety of matrices being used to establish the PK/PD profile during drug development. The final speaker of the afternoon was Liam Moran (Charles River Laboratories, OH, USA) who discussed the importance of assay format and sample analysis workflows. He focused on the choices of assay formats available to the bioanalyst with emphasis on choosing the correct format to answer the question at hand. For example, hybrid ELISA can give greater sensitivity but requires critical reagents that take time to manufacture and are costly to obtain. As an alternative, LC-MS can provide excellent selectivity but provide less sensitivity and require sample pretreatment that can be cumbersome.

The Wednesday Morning Plenary Session focused on challenges and solutions for measuring drugs in tissues and the use of surrogate matrices. In the first talk, Shane Karnik (Pyxant Labs, CO, USA) described the issues, which can be encountered during the bioanalysis of tissues and a variety of approaches to deal with them. A key concept conveyed was that the level of method qualification should be based on the type of study being supported, with regulated studies receiving as close to a complete validation as possible and discovered studies receiving an amount of qualification consistent with the level of confidence desired on the use of the data. Some of the key challenges identified by Karnik were those associated with matrix homogenization (soft vs hard tissues), matrix 
effects, establishing a realistic estimate of recovery and approaches to evidencing matrix stability (spiked matrix or incurred sample stability). Karnik concluded with three case studies which included:

- Validation of an oligonucleotide in lung and liver;

- Dilution of homogenized tissues in plasma with plasma as a surrogate matrix;

- A method to monitor 23 drugs of abuse in umbilical cord tissue. The second talk of the session was on the implications of using a surrogate matrix and was given by Stacy Ho (Sanofi, MA, USA).

In her talk, Ho described common uses of surrogate matrix to reduce the need for rare/difficult to obtain matrices, address matrix interference, achieve better LLOQ's and improve analysis efficiency. To establish surrogate matrix validity, she presented approaches and data establishing comparability in calibration curves, quality control (QC) performance, recovery and matrix effects. Methodologies to deal with endogenous analytes, including the use of stripped matrix, stable label reference standard, and a standard addition approach, were also described. In her summary, Ho stressed that qualification and validation of a surrogate matrix is time consuming and does require an amount of authentic matrix. The next presentation of the session was presented by Allysen Meymaris (Agilux Laboratories, MA, USA) on the challenges of small-molecule bioanalysis in ocular tissues during discovery and preclinical development. She began by describing the challenges associated with the anatomy of the eye and the physical differences in its various components (hard vs soft tissue). Unique sample preparation techniques for vitreous humor (aqueous), cornea (hard) and retina (soft) were provided. The importance of evaluating nonspecific binding for certain analytes was stressed along with the possible need to address stability issues (e.g., enzyme inhibitors) and the value of using surrogate species ocular matrix. The presentation concluded with two case studies demonstrating 'fit-for-purpose' method development and qualification for the bioanalysis of drugs in ocular tissues.

Wednesday afternoon consisted of two separate themes. The first looked at nanoparticle therapeutics and cutting-edge bioanalytics. The first presentation by Rafiq Islam (Celerion, NE, USA) looked at the development and optimization of an ultrasensitive immunoassay method using Amplatto technology for the measurement of protein $\mathrm{X}$ to support PK studies. He discussed the current interest and challenges in analyzing the over 600 peptides currently in preclinical and clinical development and provided several case studies utilizing the Amplatto platform for solving these challenges. The second talk on general principles, challenges and techniques for the bioanalysis of nanoparticles was presented by Ravi Viswanathan (Pfizer, CA, USA). He gave an introduction to nanoparticles and oncology, and a summary of the fractionation methods for nanoparticle bioanalysis. He outlined Pfizer's journey from discovery of bioanalysis through GLP bioanalysis and what they learned along the way relating to these analyses. He provided insight on what might be expected from upcoming US FDA and EMA guidances on existing and upcoming products in the marketplace.

Wednesday afternoon included the annual conferences 'Investigator Forum' that highlights several investigators with presentations of their research and case studies. Hyejung Park (Sanofi, MA, USA) presented targeted sphingolipid analysis by LC-MS/MS. In this presentation, she described how Sanofi established a LC-MS platform for targeted sphingolipid analysis by walking the audience through the process of developing their expertise, use of sophisticated instrumentation and fine tuning their sample extraction/preparation procedures. The second speaker was Jianyong Wang (Genentech, CA, USA) who focused on high fidelity deamidation analysis of antibody therapeutics in vivo using automated on-tip affinity capture mass spectrometry. He showed how traditional sample preparations can introduce deamination and how a new on tip protocol can mitigate artificial deamination while shortening sample preparation times. The final speaker was Katryn Allen (PRA Healthsciences, KS, USA) who discussed overcoming drug interference in a direct antidrug antibodies Assay and presented her work in overcoming interferences and the active role of acid dissociation in improving drug tolerance without limiting sensitivity.

The concluding portion for Wednesday afternoon was an overview of gene therapy and bioanalytical methods. Lilian Yengi (Bluebird Bio, MA, USA) presented the requirements and challenges related to the development of a gene therapy based CAR (chimeric antigen receptor) T-cell immunotherapy. This included a discussion on the clinical and patient challenges as well as the analytical requirements. The analytical requirements span from research based methods to clinical methods using a variety of technologies. Primarily, flow cytometry, molecular genomics and in this case, ligand-binding assays for B-cell maturation antigen. This specific gene therapy has recently received the 2017 American Society of Hemotology award. Chad Ray (Zoetis, MI, USA) followed this with a discussion on the challenges of immune-oncology based therapies. The presentation highlighted the progress of these therapies and the wide variety of analytical technologies and expertise required for their development. This 
includes high resolution mass spectrometry, flow cytometry, molecular genomics and advanced (LBA) platforms. Chad also presented their applications to investigate biological pathways and biomarkers related to treatments.

The Thursday Morning Plenary Session was an interactive session with Agency regulators looking at the relationships between the pharmaceutical industry and the FDA. Michael Day (Cardinal Health, KS, USA) gave a regulatory update on cell and gene therapy products. He summarized the past comments from International Society for Stem Cell Research 2016 annual meeting and FDA Commissioner Gottlieb's comments before Senate panel in June 2017. Day also focused on FDA approval of tissue-agnostic treatment, using an example of the pembrolizumab approval for the first cancer treatment for any solid tumors on the basis of genetic characteristics, regardless of the specific type or originating location. He discussed basket studies as one of the new trend clinical study designs and explained rationale for using a basket study for antisense oligonucleotide therapy. The second talk, focusing on the scientific rationale behind the Agency's approach to bioanalytical method validation, was presented by Jeff Moran (inVentiv Health, NE, USA). He explained scientifically why the FDA rationale makes sense for some recently issued $483 \mathrm{~s}$ and required industry compliance. The case examples included:

- Additional QCs within the established calibration range helps assure the accuracy of reported sample results;

- Freshly prepared calibrators necessary for establishing stability;

- Demonstrating reproducibility for the accuracy of sample results and incurred sample reanalysis (ISR) as an effective way to demonstrate reproducibility;

- Patient safety protected by FDA inspection procedures.

As a QA expert for regulated bioanalysis, Deborah Parker (ICON plc, NY, USA) shared her view on unexpected event investigations in Bioanalysis from her experience working at both pharmaceutical companies and contract research organizations. She described a general process for the investigation; standard operating procedure, when and how to perform the investigation, communication and reporting. She pointed out the unexpected event could be related to situations such as drug in control, failed ISR testing, multiple run failures, analyst-specific run failures, inadvertent reanalysis, repeated QC batch failures, high failure rates for IS or carryover, and chromatographic interference. As a final presentation for the conference, Brian Booth (FDA, MD, USA) shared his view on some recent frequently asked bioanalytical questions which included:

- Electronic submission requirements for bioanalytical data;

- Expectations for hybrid assays;

- Bioanalytical and PK expectations for biosimilars:

- Bioanalytical expectations for liposomal and nanoparticle drugs and ADCs.

A panel discussion was concluded after the morning presentations with lively discussion on questions collected during previous days of the conference and additional hot topics such as the progress of new FDA bioanalytical method validation guidance, validated or qualified assay for tissue sample analysis, ISR and investigation, and the need of bridging different types of assays for ADCs.

\section{Emerging technology showdowns}

In addition to the scientific presentations during the Plenary Sessions there were several presentations by contract laboratories featuring their technology to address bioanalytical challenges. The presentations included several case studies related to the technologies. The first presentation was on Tuesday afternoon with Mark Spengler (Chimera Biotec GmbH, Dortmund, Germany) presenting case studies on immunoassay PK support for biotherapeutics with special emphasis on immunotherapeutics. During the Wednesday Morning Plenary Session, there were three such presentations on the following topics:

- A workflow for bioanalytical analysis of biotherapeutics using automated affinity purification and sensitive intact protien based liquid chromatograph quad time of flight analysis by John Sausen (Agilent Technologies, WI, USA);

- Latest advances in intact and middle-down biotherapeutic protein analysis with ultrahigh resolution QTOF technologies and the latest biopharmaceuticals software - Biopharma Compass 2.0 (Jason Wood, MA, USA); 
- Life cycle management of biomarker assays as a route to improved patient outcomes (Hanna Ritzen, Mercodia $A B$, Uppsala, Sweden) on the bioanalysis of drugs in tissue during discovery and the current challenges and solutions for biomarker analysis.

The final showdown presentation was on Wednesday afternoon with Ian Moore (SCIEX, ON, Canada) discussing total antibody quantification of the antibody drug conjugate, ado-trastuzumab emtansine in rat plasma using the biologics bioanalytical solution and how a new overall survival user interface significantly increases user friendliness while providing a software package for control, data quantitation and reporting.

\section{Conclusion}

The Lands O Lakes conference stands apart from other larger international conventions by fostering the concept of sitting alongside colleagues allowing unfettered exchange of ideas and collaboration and preserves the culture that we have worked so hard to cultivate. The casual setting not only allows direct interaction and bring the community closer together but helps break down traditional notions of a quick paced, high intense conventions. Feedback was very positive. Next year's conference on 'The Next Generation of Biomarker and Bioanalytical Research' is currently being developed and will be posted on the University's website when finished by visiting our conference [1].

Financial \& competing interests disclosure

The authors have no relevant affiliations or financial involvement with any organization or entity with a financial interest in or financial conflict with the subject matter or materials discussed in the manuscript. This includes employment, consultancies, honoraria, stock ownership or options, expert testimony, grants or patents received or pending, or royalties.

No writing assistance was utilized in the production of this manuscript.

\section{Reference}

1. Annual Bioanalytical Conference (July Land O’Lakes) https://ce.pharmacy.wisc.edu/pd/bioanalyticalconf/ 
